\title{
PREGUNTAS ELABORADAS POR PROFESORES PARA EL ESTUDIO DE GRÁFICOS DE BARRAS ESTADÍSTICOS: LOS NIVELES DE LEGTURA QUE SE IDENTIFICAN EN SUS PROPUESTAS
}

\author{
QUESTIONS ADDRESSED BY TEACHERS FOR THE STUDY OF STATISTICAL BAR \\ GRAPHICS: THE READING LEVELS THAT ARE IDENTIFIED IN THEIR PROPOSALS
}

Fabiola Arévalo-Meneses

fabiola.arevalo.m@mail.pucv.cl

Pontificia Universidad Católica de Valparaíso,

Valparaíso, Chile

\author{
Julio Manzanares \\ julio.manzanares.c@mail.pucv.cl \\ Pontificia Universidad Católica de Valparaíso, \\ valparaíso, Chile
}

\section{RESUMEN}

Esta investigación de enfoque cualitativo expone los resultados de un estudio exploratorio cuyo objetivo fue mostrar el nivel de lectura de gráficos estadísticos presentes en las preguntas propuestas por docentes de Educación Matemática en Chile, ante gráficos que presentan datos de contextos contingentes en un periodo de tiempo afectado por la situación sanitaria por COVID-19 y datos que carecen de contexto. Los datos obtenidos fueron categorizados de acuerdo a los niveles de lectura de gráficos estadísticos, en donde observamos una tendencia a la elaboración de preguntas que abordan niveles básicos de lectura de gráficos estadísticos, analizando si estos tienen relación con la formación inicial de los profesores, su experiencia docente y si existe diferencia ante los gráficos presentados. Se hace relevante incorporar datos de temáticas contingentes en el estudio de gráficos estadísticos, pues promueven un nivel de lectura mayor de los datos, lo que reafirma la importancia que adquiere el contexto en el estudio estadístico, así como la necesidad de fortalecer el desarrollo profesional docente en torno a la alfabetización estadística, con el fin de formar ciudadanos críticos en una sociedad en constante cambio.

\author{
PALABRAS CLAVE: \\ Educación estadística, Formación de profesores, \\ Gráficos estadísticos, Educación en contextos \\ contingentes.
}

\section{ABSTRACT}

This research with a qualitative approach shows the results of an exploratory study whose objective was to show the reading level of statistical graphs present in the questions addressed by mathematics teachers in Chile. The graphs considered present data from contingent contexts in a period affected by the health situation of COVID-19 and the lack of context data. The data obtained were categorized according to the reading levels of statistical graphs where we observed a trend towards the elaboration of questions that address basic reading levels of statistical graphs, analyzing whether these are related to the initial formation of the teachers, their teaching experience and if there is a difference from the graphs presented. It is relevant to incorporate data on contingent topics in the study of statistical graphics, since they promote a higher reading level of the data, which reaffirms the importance of context in statistical studies, as well as the need to strengthen teacher professional development around statistical literacy, in order to train critical citizens in an ever changing society.

KEYWORDS:

Statistical education, Teacher training, Statistical

graphics, Education in contingent contexts. 


\section{Introducción}

Actualmente nos encontramos en un escenario complejo producto de la pandemia por COVID-19, que ha afectado en gran medida la vida de las personas. En este contexto, los distintos medios de comunicación nos están entregando constantemente mucha información, ante la cual, como receptores, de manera espontánea, adquirimos una visión y una percepción específica, nos formamos ideas, opiniones, juicios de valor y una postura ante la temática en cuestión. De esta manera, cobra especial importancia la interpretación y validación de información, interpelándonos como ciudadanos.

Desde la educación escolar, la formación de futuros ciudadanos capaces de insertarse en una sociedad en constante cambio resulta indispensable en el desarrollo integral de los estudiantes. Es en este escenario que la alfabetización estadística juega un rol fundamental, idea que es considerada en los planes y programas propuestos por el Ministerio de Educación de Chile (MINEDUC), los que se orientan al desarrollo de habilidades, actitudes y conocimientos en nuestros estudiantes, considerando objetivos que se enfocan en la formación integral de estos como futuros ciudadanos críticos. En Educación Matemática estos objetivos son considerados en el eje temático de Probabilidades y Estadística.

Este eje responde a la necesidad de que todos los y todas las estudiantes aprendan a realizar análisis, inferencias y obtengan información a partir de datos estadísticos. Se espera formar alumnos críticos y alumnas críticas que puedan utilizar la información para validar sus opiniones y decisiones; que sean capaces de determinar situaciones conflictivas a raíz de interpretaciones erróneas de un gráfico y de las posibles manipulaciones intencionadas que se pueden hacer con los datos. (MINEDUC, 2016, p. 102)

El gráfico estadístico es un instrumento fundamental para la organización y presentación de datos, los que se pueden describir y analizar, permitiéndonos obtener y comunicar información (Arteaga et al., 2011). Esto revela la importancia que adquiere el desarrollo de habilidades y competencias en torno al estudio de gráficos estadísticos en la educación escolar. En consecuencia, nos podemos preguntar ¿cómo se abordan los gráficos estadísticos en el aula? los profesores, ¿poseen la formación estadística que les permita abordar de manera óptima esta tarea?

Existen múltiples investigaciones en el ámbito de la educación estadística enfocadas en la lectura e interpretación de gráficos estadísticos (Aoyama y Stephens, 2003; Bertin, 1967; Curcio, 1987; Friel et al., 2001; Mautone y Mayer, 2007; Shaughnessy et al., 1996). En Contreras et al. (2017), mediante un cuestionario aplicado a futuros profesores de primaria orientado a evaluar aspectos relevantes de la cultura estadística, se observó que existe una escasa comprensión gráfica, concluyendo acerca de la importancia que adquiere la revisión de los programas de formación de profesores. En el mismo sentido, Espinel (2007) muestra resultados de dos investigaciones anteriores, también en futuros profesores de primaria, una de ellas referente a construcción y evaluación de histogramas y otra sobre representaciones de distribución de datos, donde se observan los errores frecuentes que cometen en la construcción de gráficas y dificultades que presentan en el razonamiento frente a distintas representaciones de distribuciones de datos. Por otra parte, en Arteaga et al. (2011) se reúnen los aspectos más importantes de investigaciones referentes a las competencias que se requieren para la construcción y lectura de tablas y gráficos estadísticos, destacando la necesidad de que los profesores estén bien preparados para así formar ciudadanos estadísticamente cultos, lo que se puede lograr mejorando la cultura estadística en futuros profesores a través de su formación. Otra investigación realizada por Estrella et al. (2015) considera tres elementos fundamentales dentro de la educación estadística, entre los cuales se encuentra la comprensión gráfica. En este estudio se aplicó un cuestionario a profesores de educación primaria en Chile y a sus estudiantes, cuyos resultados mostraron un muy bajo desempeño en la lectura interpretativa de gráficos estadísticos tanto en profesores como en estudiantes, haciendo énfasis en la necesidad de que los profesores se enfrenten a situaciones de incerteza en el estudio estadístico.

Rodríguez y Sandoval (2012) indagaron en el nivel de lectura de gráficos estadísticos en 47 profesores de primaria y 44 futuros profesores de primaria en Chile, comparando ambos grupos en relación a la habilidad de codificación y decodificación de información gráfica, evidenciando un bajo nivel tanto en profesores como en futuros profesores de primaria.

Por su parte, Díaz-Levicoy et al. (2015) analizaron los tipos de gráficos y sus niveles de lectura presentes en 12 textos de educación primaria en Chile, en donde la mayoría eran gráficos de barras y pictogramas, siendo "leer dentro de los datos" el nivel de lectura más frecuente. Es así que se observa la necesidad de incorporar actividades que contemplen niveles de lectura de datos más profundos en cursos superiores. De esta forma, surge de manera natural la preocupación de indagar en relación a cómo se enseña estadística en la educación escolar, cuáles son las limitaciones que presenta el docente al momento de enseñar estadística, y puntualmente cómo aborda el estudio de gráficos estadístico con sus estudiantes, qué habilidades y competencias espera desarrollar en ellos. Sin dudas es una situación interesante de ser investigada, pues es a través de la alfabetización estadística que los estudiantes podrán ser ciudadanos efectivos en la sociedad de la información (Pino y Estrella, 2012).

Considerando el contexto de pandemia producto de COVID-19, se han llevado a cabo varias investigaciones 
desde esta temática. Alsina et al. (2020) describen experiencias de aprendizaje relacionadas con la pandemia, tomando como referencia investigaciones anteriores que declaran la importancia de considerar temáticas contextuales que aporten significado al estudio estadístico. Del mismo modo, Coob y Moore (1997) señalan que los datos son números que se encuentran en un contexto, lo que les da significado. Por otra parte, un estudio reciente (Vásquez, 2021), en el mismo marco de la emergencia sanitaria por COVID-19, analizó las preguntas que plantearon 48 futuros profesores de educación primaria en Chile para la comprensión de gráficos y el desarrollo de competencias de sostenibilidad en sus estudiantes. Los resultados mostraron que la mayoría de las preguntas planteadas por los futuros profesores se encuentran en los niveles 1 y 2 ("leer los datos" y "leer dentro de los datos"), que se refieren a preguntas que no requieren de un análisis profundo de los datos, indicando que puede deberse a que las preguntas elaboradas por futuros profesores van dirigidas a estudiantes de primaria, motivo por el cual el nivel de dificultad no puede ser mayor, o bien que los futuros profesores participantes no son capaces de plantear preguntas que consideren niveles de lectura más profundos. Por otra parte, señalan que solo dos de los profesores plantearon preguntas características del nivel 4 "leer detrás de los datos", que son aquellas que contribuyen al desarrollo del pensamiento crítico y comprensión del contexto en que se encuentra la información.

Si bien el presente estudio se desarrolló sin conocer la investigación mencionada anteriormente, dada su reciente publicación, podemos observar que considera elementos comunes a los que se abordan en esta investigación, lo que nos brinda la posibilidad de poder contrastar y comparar en el análisis de los datos. Ahora bien, el presente estudio considera a profesores de educación primaria y profesores de Matemáticas, los cuales ya terminaron su formación inicial docente. Por otra parte, se consideran gráficos de barras estadísticos que, de acuerdo a los datos que presentan, no necesariamente tienen relación con la crisis sanitaria vivida actualmente, sin embargo, no se hace referencia al respecto con el fin de no interferir en la interpretación de la información por parte de los profesores.

Considerando los antecedentes expuestos, el objetivo de este estudio es indagar en los niveles de lectura de gráficos estadísticos presentes en preguntas elaboradas por profesores que actualmente realizan docencia, dirigidas a sus estudiantes para el estudio de gráficos de barras estadísticos. Y a la vez, identificar si la formación inicial y experiencia de los profesores, así como la temática relacionada con los datos presentados en el gráfico, inciden en las preguntas que realizan.

\section{Marco de referencia}

La base de esta investigación radica en el análisis de los niveles de lectura e interpretación de gráficos estadísticos, comprendiendo que la lectura comienza con una identificación de lo externo (barras, título, etc.), y luego lo interno (variables, significados de las variables, escalas, etc.), distinguiendo tres niveles de lectura de gráficos: N1: Extracción de los datos; N2: Extracción de las tendencias, y N3: Análisis de la estructura de los datos (Bertin, 1967).

Por otra parte, Curcio (1987) señala que para la lectura y construcción de gráficos se debe comprender tres aspectos que son característicos en ellos:

- Las expresiones o palabras.

- Lo matemático que se subyace.

- El convenio de construcción de gráficos.

Más tarde, por la necesidad de la comprensión de todos estos aspectos, Curcio (1989) estableció niveles de lectura, ampliando la definición de Bertin (1967). Estos son:

L1: Leer los datos: cuando se realiza una lectura literal de algún dato representado en el gráfico estadístico.

L2: Leer dentro de los datos: cuando se realiza una lectura de un dato del gráfico que no se encuentra de forma explícita en él, y que requiere de alguna operación matemática (adición, promedio, etc.) para encontrarlo.

L3: Leer más allá de los datos: cuando se obtiene una información que no se presenta directamente en el gráfico, y no es posible encontrarla por medio de cálculos u operaciones, infiriendo dicho dato.

Con el pasar del tiempo, y por la necesidad que se fue presentando en la investigación respecto del análisis y comprensión de los gráficos estadísticos, Friel et al. (2001) ampliaron nuevamente lo propuesto por Curcio, agregando una clasificación más. Esta es:

L4: Leer detrás de los datos: cuando se realiza una valoración crítica de la información presentada en el gráfico estadístico sobre la recogida de datos, su organización o las conclusiones. A ello se suma el conocimiento del contexto en el que se extraen los datos del gráfico.

En base a lo planteado por Curcio (1989) y Friel et al. (2001), esta investigación utiliza los niveles de lectura de gráficos estadísticos de los autores señalados, para analizar el enfoque de las preguntas realizadas por docentes de Matemática en base a gráficos estadísticos.

\section{Metodología}

La presente investigación se realiza mediante una metodología de tipo cualitativa (Hernández et al., 2015). A través del análisis de contenido (Krippendorff, 2013), se busca indagar en las preguntas que proponen profesores de Educación Matemática, dirigidas a sus 
estudiantes, para el estudio de dos gráficos de barras estadísticos.

\subsection{Caracterización de los sujetos de estudio}

Los sujetos de estudio son docentes de enseñanza de la Matemática que se encuentran realizando sus funciones pedagógicas en establecimientos escolares de la quinta región de Valparaíso, Chile. En total, 22 docentes respondieron el instrumento, entre los que se encuentran profesores cuya formación inicial es profesor de educación primaria, quienes realizan docencia en educación primaria. Y profesores de formación inicial de pedagogía en Matemáticas, quienes realizan docencia en educación secundaria.

\subsection{Diseño del instrumento}

El instrumento que se utilizó para recoger los datos fue un formulario, el cual por la contingencia que se suscita a nivel mundial (pandemia COVID-19), se tuvo que realizar de manera online, por medio de la plataforma Google (Google Forms), a través de la cual se buscó en primera instancia caracterizar a los sujetos de investigación, consultando por su formación inicial y los años de experiencia que poseen en el sistema escolar. Posteriormente se les presentó dos gráficos de barras con la finalidad de conocer qué tipo de preguntas dirigidas a sus estudiantes realizarian en relación a estos.

El primer gráfico (G1) presenta información sobre la cantidad de latas recolectadas por una comunidad, en donde se puede observar la cantidad recolectada por hombres y por mujeres en cada año, desde el año 2014 al año 2017 (ver Figura 1).

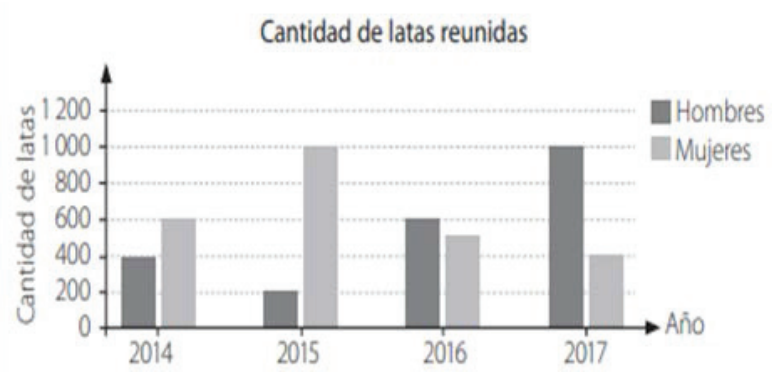

Figura 1. Gráfico (G1), cantidad de latas reunidas por una comunidad. Nota. Imagen de recursos de la unidad para $6^{\circ}$ básico

(Curriculumnacional.mineduc.cl).

El gráfico G2 corresponde a una boleta de consumo de agua de un hogar ubicado en la quinta región de Valparaíso, en que se muestra el consumo de agua desde abril de 2019 a abril de 2020 (ver Figura 2). Cabe destacar que es durante este periodo de tiempo que Chile entra en estado de emergencia sanitaria por la presencia de COVID-19, específicamente en marzo del año 2020. Ahora bien, el gráfico se muestra tal y como se puede observar en la Figura 2, es decir, no se indican las posibles causas del aumento en el consumo de los últimos meses, pudiendo deberse a diferentes factores. El propósito es no interferir en las interpretaciones y el sentido que el profesor puede dar a la información que entrega el gráfico, lo que puede verse reflejado en las preguntas que elabora para sus estudiantes.

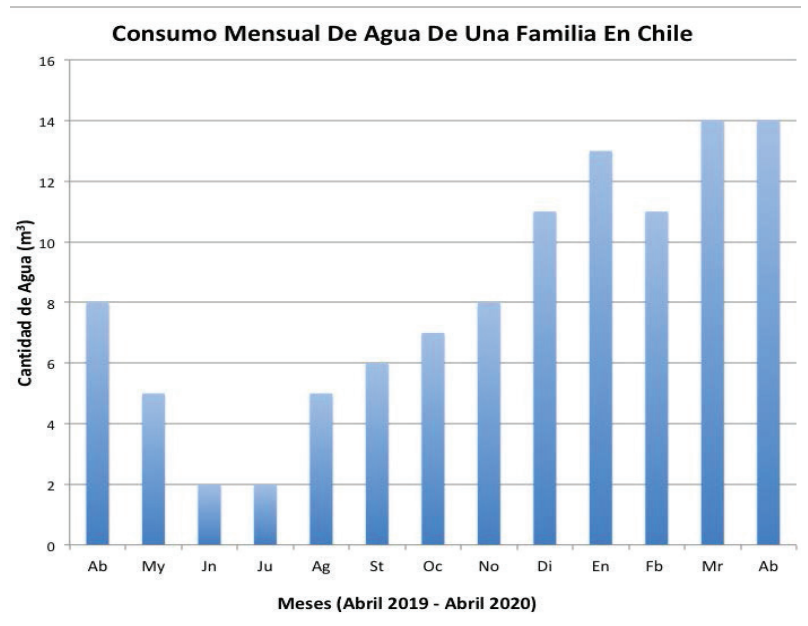

Figura 2. Gráfico 2 (G2), consumo mensual de agua de una familia en Chile. Nota. Boleta de Esval de un departamento en la V región, Chile.

Para cada uno de los gráficos (G1 y G2) se les pidió a los profesores que diseñaran preguntas que ellos plantearían a sus estudiantes para el estudio de estos.

\subsection{Recogida de datos}

Dadas las actuales medidas de seguridad que se han tomado en el marco de la crisis sanitaria por COVID-19, se consideró la mejor alternativa en cuanto a herramientas tecnológicas que nos permiten comunicarnos de forma efectiva y que a la vez son de fácil acceso para los profesores, de este modo, se envió un formulario vía correo electrónico. Esto permitió contar de forma inmediata con los datos una vez que los profesores respondieran el cuestionario. De esta manera se obtienen los siguientes resultados (ver Tabla 1). 
Tabla 1. Caracterización de los datos obtenidos Nota. Elaboración propia.

\begin{tabular}{|c|c|}
\hline \multicolumn{3}{|c|}{ Cantidad de preguntas por gráficos } \\
\hline G1 & G2 \\
\hline 61 & 55 \\
\hline \multicolumn{3}{|c|}{ Total de preguntas: 116} \\
\hline
\end{tabular}

\begin{tabular}{|c|c|}
\hline \multicolumn{2}{|c|}{ Profesores por formación inicial } \\
\hline Educación básica & Pedagogía en Matemática \\
\hline 6 & 16 \\
\hline \multicolumn{2}{|c|}{ Total de profesores: 22} \\
\hline
\end{tabular}

\begin{tabular}{|c|c|c|c|}
\hline \multicolumn{4}{|c|}{ Profesores por experiencia laboral } \\
\hline $\begin{array}{c}\text { Entre 0 y } 3 \\
\text { años }\end{array}$ & $\begin{array}{c}\text { Entre } 3 \text { y } 7 \\
\text { años }\end{array}$ & $\begin{array}{c}\text { Entre 7 y 12 } \\
\text { años }\end{array}$ & $\begin{array}{c}\text { Más de 12 } \\
\text { años }\end{array}$ \\
\hline 4 & 6 & 6 & 6 \\
\hline \multicolumn{4}{|c|}{ Total de profesores: 22 } \\
\hline
\end{tabular}

\subsection{Categorías de análisis}

Considerando los niveles de lectura de gráficos propuestos por Friel et al. (2001), se definen en la Tabla 2 las siguientes categorías para clasificar las preguntas realizadas por los profesores en ambos gráficos propuestos, de acuerdo a la intencionalidad que se puede apreciar en las pregunta realizadas en relación a lo que motivan en el estudiante.

Tabla 2. Categoría de análisis

Nota. Elaboración propia.

\begin{tabular}{|l|l|l|}
\hline Sigla & Categoria & Definición \\
\hline L1 & $\begin{array}{l}\text { Preguntas de } \\
\text { nivel L1 de } \\
\text { lectura de } \\
\text { gráficos. }\end{array}$ & $\begin{array}{l}\text { La pregunta induce a la lectura } \\
\text { literal de datos. }\end{array}$ \\
\hline L2 & $\begin{array}{l}\text { Preguntas de } \\
\text { nivel L2 de } \\
\text { lectura de } \\
\text { gráficos. }\end{array}$ & $\begin{array}{l}\text { La pregunta induce a realizar una } \\
\text { lectura de un dato del gráfico que } \\
\text { no se encuentra de forma explícita } \\
\text { en él, y que requiere de alguna } \\
\text { operación matemática (adición, } \\
\text { promedio, etc.) para encontrarlo. }\end{array}$ \\
\hline L3 & $\begin{array}{l}\text { Preguntas de } \\
\text { nivel L3 de } \\
\text { lectura de } \\
\text { gráficos. }\end{array}$ & $\begin{array}{l}\text { La pregunta induce a extraer } \\
\text { información que no se presenta } \\
\text { directamente en el gráfico, y no es } \\
\text { posible encontrarla por medio de } \\
\text { cálculos u operaciones, infiriendo } \\
\text { dicho dato. }\end{array}$ \\
\hline L4 & $\begin{array}{l}\text { Preguntas de } \\
\text { nivel L4 de } \\
\text { lectura de } \\
\text { gráficos. }\end{array}$ & $\begin{array}{l}\text { La pregunta induce a realizar } \\
\text { una valoración crítica de la } \\
\text { información presentada en el } \\
\text { gráfico (sobre la recogida de } \\
\text { datos, su organización o las } \\
\text { conclusiones). A ello se suma el } \\
\text { conocimiento del contexto en } \\
\text { el que se extraen los datos del } \\
\text { gráfico. }\end{array}$ \\
\hline
\end{tabular}

\section{Resultados}

A partir de los datos obtenidos se presentan a continuación los resultados en relación a las preguntas elaboradas al comparar ambos gráficos respecto de la formación inicial y de la experiencia docente.

\subsection{Resultados en relación a los gráficos presentados}

La Figura 3 presenta el resultado en relación a la clasificación de las preguntas por gráficos.

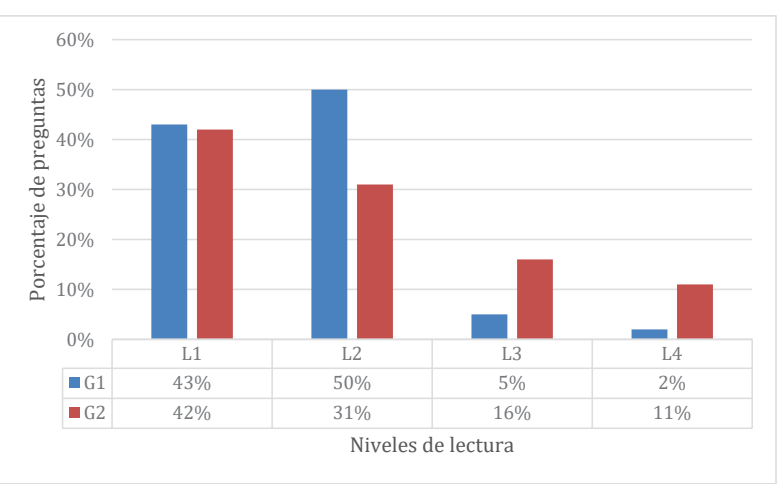

Figura 3. Clasificación de las preguntas por gráficos

Nota. Elaboración propia.

En la Figura 3 se representa la relación entre los niveles de lectura de gráfico que abordan las preguntas realizadas y el gráfico al que pertenecen. Podemos observar que la mayoría de las preguntas elaboradas por los docentes se encuentran en el nivel L1 (leer los datos) y L2 (leer dentro de los datos) de lectura de gráficos de la taxonomía de Curcio (1987), que en conjunto corresponden a un $83 \%$ de las preguntas (ver ejemplos en Tabla 3). Mientras que una cantidad significativamente menor (10\%) se encuentra en el nivel L3 (leer más allá de los datos) y tan solo un $6 \%$ corresponde a preguntas del nivel L4 (leer detrás de los datos), propuesto por Friel et al. (2001).

Tabla 3. Ejemplos de preguntas de niveles L1 y L2 Nota. Preguntas extraidas de datos obtenidos.

\begin{tabular}{|l|l|l|}
\hline Gráfico & Nivel & Pregunta realizada por profesor (P) \\
\hline G1 & L1 & $\begin{array}{l}\text { ¿En qué año se logró la mayor } \\
\text { recolección de latas por parte de las } \\
\text { mujeres? (P13) }\end{array}$ \\
\hline G2 & L2 & $\begin{array}{l}\text { ¿Cuál es la diferencia de consumo de } \\
\text { agua entre los meses de abril 2019 y } \\
\text { abril 2020? (P8) }\end{array}$ \\
\hline
\end{tabular}

Considerando las preguntas realizadas de acuerdo al gráfico 1 (G1) y al gráfico 2 (G2), podemos notar que en los niveles L1 y L2 se presenta una diferencia 
considerable, en tanto que en G1 estos niveles concentran el $93 \%$ de las preguntas, mientras que en G2, un 73\%. Por otra parte, en los niveles L3 y L4 existe una gran diferencia entre los gráficos G1 (7\%) y G2 (27\%) (ver ejemplos en Tabla 4).

Tabla 4. Ejemplos de preguntas niveles L 3 y L 4 Nota. Preguntas extraídas de datos obtenidos.

\begin{tabular}{|l|l|l|}
\hline Gráfico & Nivel & Pregunta realizada por profesor (P) \\
\hline G1 & L3 & $\begin{array}{l}\text { ¿Qué puedes inferir respecto de la } \\
\text { recolección de latas realizadas por las } \\
\text { mujeres y los hombres durante los 4 } \\
\text { años? (P21) }\end{array}$ \\
\hline G2 & L4 & $\begin{array}{l}\text { ¿Qué circunstancia crees que existieron } \\
\text { durante el periodo de mayor consumo } \\
\text { de agua en la familia? (P15) }\end{array}$ \\
\hline
\end{tabular}

De esta manera, podemos ver que la mayoría de las preguntas de los niveles L1 y L2 pertenecen a G1 (recolección de latas), y la mayoría de las preguntas de los niveles L3 y L4 se encuentran en G2 (consumo de agua).

\subsection{Resultados en relación a la formación inicial}

La Figura 4 presenta el resultado en relación a la clasificación de las preguntas de acuerdo a la formación inicial.

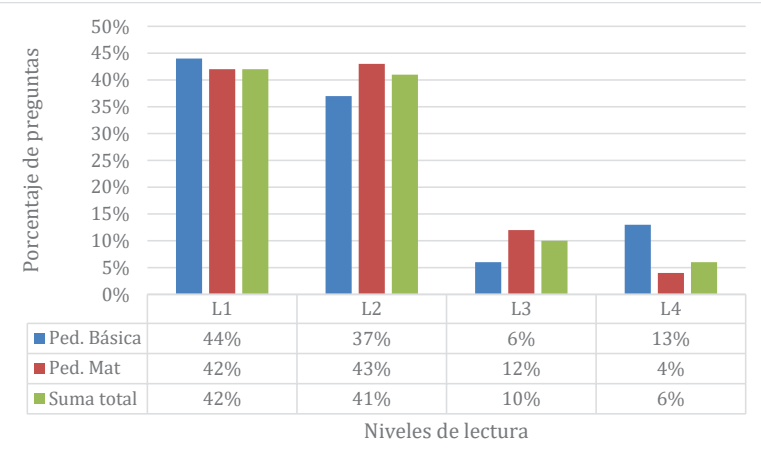

Figura 4. Clasificación de las preguntas por formación inicial. Nota. Elaboración propia.

La Figura 4 muestra la relación entre la formación inicial de los docentes y los niveles de lectura de gráficos presentes en sus preguntas, en los cuales podemos observar que no existe mayor diferencia porcentual entre la cantidad de preguntas realizadas en los niveles L1 y L2 por docentes cuya formación inicial es pedagogía en educación primaria (44\% y $38 \%$, respectivamente), y las realizadas por profesores cuya formación inicial es pedagogía en Educación Matemática ( $42 \%$ y $43 \%$, respectivamente). Por otra parte, la mayor cantidad de preguntas del nivel L3 son realizadas por profesores de educación secundaria $(12 \%)$, en comparación a educación primaria (6\%). Ahora bien, la relación se invierte en el nivel L4, siendo mayor la cantidad de preguntas realizadas por profesores de educación primaria (13\%), mientras que en educación secundaria es tan solo un $4 \%$. Sin embargo, no podemos inferir respecto de estas diferencias en el nivel L3 y L4 en relación a la formación docente dado que la cantidad de preguntas de estos niveles fueron muy escasas.

\subsection{Resultados en relación a la experiencia docente}

La Figura 5 presenta el resultado en relación a la clasificación de las preguntas de acuerdo a la experiencia docente.

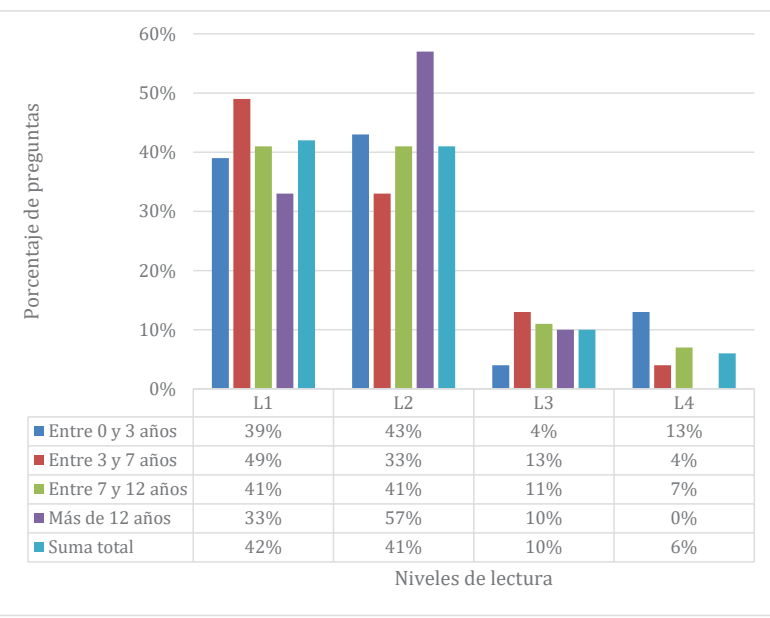

Figura 5. Clasificación de las preguntas por años de experiencia docente. Nota. Elaboración propia.

De la relación entre la experiencia laboral y el nivel de lectura de gráficos de las preguntas realizadas, se observa que en todos los rangos de años de experiencia docente se concentra al menos el $83 \%$ de las preguntas en los niveles L1 y L2, y tan solo un $16 \%$ de las preguntas se encuentran en los niveles L3 y L4, siendo en el rango de 0 a 3 años de experiencia el que presenta mayor cantidad de preguntas del nivel L4 (13\%), mientras que, en este mismo nivel de lectura de gráficos, los docentes que poseen 12 o más años de experiencia ( $27 \%$ de los encuestados), no realizan ninguna pregunta. Así también, podemos notar en este mismo rango de experiencia, que más de la mitad de las preguntas (57\%) son preguntas pertenecientes al nivel $\mathrm{L} 2$.

Podemos afirmar de esta manera que, en todos los rangos de experiencia docente, se mantiene la tendencia a realizar una gran cantidad de preguntas de nivel L1 y L2, que apuntan a la descripción e interpretación de los datos, mientras que, entre más años de experiencia docente tengan los profesores, la cantidad de preguntas realizadas en los niveles $L 3$ y $L 4$ 
tienden a disminuir y a desaparecer, que son aquellas que buscan realizar inferencias y una valoración crítica de los datos.

\section{Análisis}

De acuerdo al análisis de los datos obtenidos, constatamos que efectivamente las preguntas elaboradas por los profesores se concentran en los niveles de lectura L1 (leer los datos) y L2 (leer dentro de los datos), de acuerdo a la taxonomía de lectura de gráficos (Curcio, 1987; Friel et al., 2001). Este resultado concuerda con lo observado por Alsina et al. (2020), Contreras et al. (2017), Estrella et al. (2015) y recientemente por Vásquez (2021) en futuros profesores de educación primaria, de los cuales muy pocos alcanzan los niveles más altos en lectura de gráficos. Por otra parte, en una investigación en que se analizaron textos escolares de educación primaria en Chile y España, se observó que el nivel más frecuente es "leer dentro de los datos" (Díaz-Levicoy et al., 2015), lo que podría estar relacionado con la tendencia de los profesores a presentar preguntas enfocadas a obtener datos desde la lectura de las etiquetas y valores presentes en el gráfico así como la comparación de datos, y no a la valoración crítica de la información que se puede inferir desde el gráfico.

En relación a la experiencia y la formación inicial docente, no es posible observar que tenga relación directa con el nivel de lectura de gráficos presente en las preguntas. No obstante, un caso particular fue el de aquellos profesores con 12 o más años de experiencia, de los cuales ninguno realizó preguntas del nivel L4 "leer detrás de los datos". Es posible que dicho suceso pueda estar relacionado con las áreas de conocimiento que los profesores han abordado durante sus años de experiencia docente, pues los datos obtenidos no nos proporcionan información concreta en relación a cuánto tiempo de su vida profesional han dedicado a la enseñanza de la estadística, por lo que no podemos conjeturar al respecto. Pino y Estrella (2012) señalan que la formación académica de los docentes es débil y que sus experiencias en educación estadística son mínimas. Considerando lo anterior, resulta necesario poder indagar en la experiencia de los profesores, particularmente en enseñanza de estadística durante sus años en ejercicio, así como indagar y profundizar en la formación profesional. Sin embargo, más allá de este caso particular, de manera transversal se destaca la casi inexistencia de preguntas del nivel L4, lo que también se observa en Vásquez (2021) con futuros profesores de primaria, en donde ninguno de ellos propuso preguntas de este nivel. Sin dudas esto resulta preocupante y se transforma en un elemento que debe ser considerado al momento de diseñar programas de desarrollo profesional docente.

De acuerdo a los resultados de esta investigación, en profesores de educación básica si bien la mayoría de sus preguntas son de niveles L1 y L2, lo que está acorde a lo establecido por las bases curriculares, creemos necesario incorporar preguntas de los niveles L3 y L4 en los últimos cursos de la enseñanza básica, tal como también lo afirman Díaz-Levicoy et al. (2015). Lo que observamos en preguntas de los profesores de educación secundaria es similar a lo ocurrido en educación primaria, es decir, la mayoría de las preguntas son de niveles L1 y L2, y se alejan de lo expresado en las bases curriculares en el eje de Datos y Azar, pues en este ciclo se espera que los estudiantes realicen inferencias y valoración crítica de la información a partir de datos estadístico, niveles L3 y L4 (MINEDUC, 2016).

Por otra parte, en relación a los gráficos presentados a los docentes, notamos que existe una situación particular en el gráfico G2 (consumo de agua), pues es en este donde se concentra la mayoría de las preguntas de los niveles L3 y L4. El gráfico G2 presenta el consumo de agua de una vivienda en la ciudad de Valparaíso, en el periodo de abril de 2019 hasta abril de 2020. Desde marzo del 2020 Chile se ha visto afectado por la crisis sanitaria por COVID-19, situación que ha provocado un incremento en los consumos básicos del hogar, tal como se indica en el estudio publicado por el Centro del Agua para Zonas Áridas y Semiáridas de América Latina y el Caribe (CAZALAC) en Mansilla et al. (2020), que hace referencia a la demanda hídrica en Chile ante la pandemia. El estudio revela que el consumo de agua potable en un hogar aumenta al $69,3 \%$ en relación al consumo habitual, durante el periodo afectado por la crisis sanitaria e hídrica del país. Este aumento podría deberse a las medidas que se han tomado para prevenir el contagio de COVID-19, tales como mayor frecuencia de higiene personal, del hogar y de los alimentos. Es así, que en el gráfico G2, se presentan datos en relación a una temática de contingencia mundial, por tanto, de gran interés y preocupación, que afecta hoy en día directamente la vida de las personas.

Es importante recordar que el gráfico fue presentado a los profesores sin realizar ninguna observación en relación al contexto ni a las posibles causas del aumento en el consumo de agua, las que podrían ser múltiples y diversas. Esto nos lleva a pensar que el hecho de estar viviendo una situación tan particular como la crisis sanitaria por COVID-19, parece predisponer de manera casi innata al observador del gráfico, de tal modo que advierte el aumento en el consumo y lo relaciona directamente con lo que se vive en tiempos de pandemia. Podríamos decir que la elaboración de preguntas de los niveles L3 y L4 que promueven un análisis crítico de la información se ve motivada en parte por los conocimientos adquiridos por el profesor de acuerdo a su propia experiencia personal, desde donde da sentido a la información. De cierto modo, el profesor agrega conocimiento referente al gráfico, lo que se puede apreciar en la intencionalidad de las preguntas que elabora. Es así como el conocimiento del profesor en relación a la información y contexto de los datos que se presentan en el gráfico, resultan un factor muy importante al momento en que el profesor realiza preguntas orientadas al estudio de gráficos estadísticos. Si bien, necesitamos más datos que nos 
permitan conjeturar al respecto, existen estudios que destacan la relevancia que adquiere el contexto en la enseñanza de la estadística. Coob y Moore (1997), así como Pino y Estrella (2012), expresan que en la enseñanza de la estadística el contexto en que se presentan los datos es de suma importancia para la alfabetización estadística. Del mismo modo, Batanero et al. (2013) relacionan la cultura estadística y el razonamiento estadístico como las dos componentes que dan sentido al estudio estadístico, indicando que sin considerar el contexto donde se encuentran los datos, se pierde dicho sentido. Esta idea es apoyada en Alsina et al. (2020) y en Vásquez (2021), quienes en recientes investigaciones presentan una serie de experiencias contextualizadas con datos de COVID-19, en que concluyen que mediante estas actividades los estudiantes desarrollan la capacidad de reflexionar y ser críticos ante la información.

\section{Conclusiones}

En este estudio se observó que, independiente de la formación inicial del profesor, de la experiencia docente y de los datos que se presentan en el gráfico de estudio, la gran mayoría de las preguntas que los profesores realizan ante el estudio de gráficos estadísticos se encuentran en los nivel de lectura L1 y $L 2$, a las que se puede dar respuesta desde la lectura literal de los datos y de interpretación de datos que se fundamentan en la comparación y cálculos a partir de los datos, siendo escasa y casi nula la propuesta de preguntas que propongan al estudiante realizar un nivel más profundo de lectura de gráficos, que apunten a realizar predicciones e inferencias, así como un análisis crítico de la información. Esto presenta un gran desafío para las instituciones de formación inicial y continua de profesores, que promueven la adquisición de conocimientos de la disciplina y su didáctica específica, con el fin de formar ciudadanos estadísticamente cultos y comprometidos socialmente (Arteaga et al., 2011).

No obstante, podemos decir que esto no es suficiente, pues hemos podido observar la importancia que adquiere el conocimiento que el profesor tenga del contexto en relación a los datos presentes en el gráfico, lo que le permite intensionar un análisis más profundo y crítico de la información. Esto presenta un desafío importante para el docente, quien debe mantenerse constantemente informado en relación a temáticas de interés colectivo y que sean significativas para sus estudiantes. En consecuencia, no se puede perder de vista lo importante que es incorporar situaciones contextualizadas, que sean significativas para los estudiantes, ante lo cual también es necesario que el profesor tenga conocimiento en relación a los datos, así como del contexto en que estos se presentan.

El factor cultura adquiere protagonismo. Ambos, profesor y estudiante, están inmersos en un entorno sociocultural que forman parte de su identidad. El profesor no es un sujeto ajeno en el proceso de formación de sus estudiantes, su propia experiencia se hace presente al momento de guiarlos hacia la valoración y el análisis crítico de información en donde el contexto actúa como un factor mediador en el estudio estadístico no solo para el estudiante, sino también para el profesor, quien también debe continuar en constante formación.

\section{Agradecimientos}

Este trabajo fue realizado con el apoyo de la Beca de Manutención 2021 de la Pontificia Universidad Católica de Valparaíso.

Agradecimiento especial a la Dra. Elisabeth Ramos Rodríguez por sus revisiones y motivaciones para culminar este trabajo. 


\section{Referencias}

Alsina, Á., Vásquez, C., Muñiz-Rodríguez, L., y Rodríguez-Muñiz, L. J. (2020). ¿Cómo promover la alfabetización estadística y probabilística en contexto? Estrategias y recursos a partir de la COVID-19 para Educación Primaria. Épsilon-Revista de Educación Matemática, 104, 99-125.

Aoyama, K., y Stephens, M. (2003). Graph interpretation aspects of statistical literacy: A Japanese perspective. Mathematics Education Research Journal, 15(3), 207225. https://doi.org/10.1007/BF03217380

Arteaga, P., Batanero, C., Cañadas, G., y Contreras, M. (2011). Las tablas y gráficos estadísticos como objetos culturales. Números. Revista de Didáctica de las Matemáticas, 76, 55-67.

Batanero, C., Díaz, C., Contreras, J. M., y Roa, R. (2013). El sentido estadístico y su desarrollo. Números, 83, 7-18.

Bertin, J. (1967). Sémiologie graphique. Gauthier-Villars.

Cobb, G. W., y Moore, D. S. (1997). Mathematics, Statistics, and Teaching. The American Mathematical Monthly, 104(9), 801-823. https://doi.org/10.1080/000 29890.1997.11990723

Contreras, J., Molina-Portillo, E., Godino, J. D., y Batanero, C. (2017). Construcción de un cuestionario para evaluar la interpretación crítica de gráficos estadísticos por futuros profesores. En J. M. Muñoz, A. Arnal-Bailera, P. Beltrán-Pellicer, M. L. Callejo, y J. Carrillo (Eds.), Investigaciones en Educación Matemática XXI (207-216). Universidad de Zaragoza.

Curcio, F. R. (1987). Comprehension of Mathematical Relationships Expressed in Graphs. Journal for Research in Mathematics Education, 18(5), 382-393. https://doi. org/10.5951/jresematheduc.18.5.0382

Curcio, F. R. (1989). Developing Graph Comprehension. Elementary and Middle School Activities. National Council of Teachers of Mathematics, Inc., 1906 Association Drive, Reston, VA 22091.

Díaz-Levicoy, D., Arteaga, P., y Batanero, C. (2015). Gráficos estadísticos y niveles de lectura propuestos en textos chilenos de educación primaria. En C. Fernández, M. Molina y N. Planas (Eds.), Investigaciones en Educación Matemática XIX (pp. 229-238). SEIEM.

Espinel, M. (2007). Construcción y Razonamiento de Gráficos Estadísticos en Formación de Profesores. Investigación en Educación Matemática, 11, 99-119.

Estrella, S., Olfos, R., y Mena-Lorca, A. (2015). El conocimiento pedagógico del contenido de estadística en profesores de primaria. Educação e Pesquisa, 41(2), 477-493. https://doi.org/10.1590/s151797022015041858.
Friel, S. N., Curcio, F. R., y Bright, G. W. (2001). Making Sense of Graphs. Journal for Research in Mathematics Education, 32(2), 124-158. https://doi. org/10.23077749671

Hernández, R., Fernández, C., y Baptista, P. (2014). Metodología de la Investigación (6.a edición). McGraw Hill.

Krippendorff, K. (2013). Content analysis: an introduction to its methodology. SAGE.

Mansilla, G., Soto, M., y Vivanco, C. (2020). COVID-19: Implicancias y repercusiones en la seguridad hidrica. Centro Regional del Agua para Zonas Áridas y Semiáridas de América Latina y el Caribe, CAZALAC.

Mautone, P. D., y Mayer, R. E. (2007). Cognitive Aids for Guiding Graph Comprehension. Journal of Educational Psychology, 99(3), 640-652. https://doi. org/10.1037/0022-0663.99.3.640

Ministerio de Educación de Chile. (2016). Bases curriculares vigentes. Unidad de Curriculum y Evaluación.

Pino, G. D. E. L., y Estrella, S. (2012). Educación estadística: relaciones con la matemática. Revista de Investigación Educacional Latinoamericana, 49(1), 5364. http://dx.doi.org/10.7764/PEL.49.1.2012.5

Rodríguez, F., y Sandoval, P. (2012). Descodificación de tablas y gráficos estadísticos: Un estudio comparativo en profesores y alumnos de pedagogía en Enseñanza Básica. Avaliação: Revista da Avaliação da Educação Superior, 17(1), 207-235. https://doi.org/10.1590/S141440772012000100011

Shaughnessy, J., Garfield, J., y Greer, B. (1996). Data Handling. En A. Bishop, K. Clements, C. Keitel, J. Kilpatrick y C. Laborde (Eds.), International Handbook of Mathematics Education. Kluwer International Handbooks of Education (vol. 4, pp. 205-237). Springer. https://doi. org/10.1016/B978-0-12-375142-3.10006-9

Vásquez, C. (2021). Comprensión y Uso Docente de Gráficos Estadísticos por Futuros Profesores para Promover Competencias para la Sostenibilidad. Paradigma, 41(e1), 165-190. https://doi.org/10.37618/ PARADIGMA.1011-2251.2021.p165-190.id1022 